\title{
Study of Experimental Simple Pendulum Approximation Based on Image Processing Algorithms
}

\author{
Mohammed Y. Kamil \\ Department of Physics, College of Sciences, AL-Mustansiriyah University, Iraq \\ Tel: 964-770-257-5673 E-mail: m80y98@yahoo.com \\ Ali Abid D. Al-Zuky \\ Department of Physics, College of Sciences, AL-Mustansiriyah University, Iraq \\ Tel: 964-770-273-2705Ｅ-mail:dr.alialzuky@yahoo.com \\ Radhi Sh. Al-Tawil \\ Department of Physics, College of Education, AL-Mustansiriyah University, Iraq \\ Tel: 964-780-213-4935Ｅ-mail: dr.radhialtawil@yahoo.com
}

Received: December 6, 2010 Accepted: December 22, 2010 doi:10.5539/apr.v3n1p29

\begin{abstract}
In this study we used image processing algorithms to determine pendulum ball motion and present an approach for solving the nonlinear differential equation that governs its movement. This formulae show an excellent agreement with the exact period calculated with the use of elliptical integrals, and they are valid for both small and large amplitudes of oscillation.
\end{abstract}

Also, we reveal some interesting aspects of the study simple pendulum or procedures for determining analytical approximations to the periodic solutions of nonlinear differential equations.

Keywords: Simple pendulum, Large amplitude, Exact period, Image processing, Nonlinear equation

\section{Introduction}

The simple pendulum is one of the most popular examples analyzed in the textbooks and undergraduate courses in physics and it is perhaps the most investigated oscillatory motion in physics [Baker G, 2005]. Many nonlinear phenomena in the real world are governed by pendulum-like differential equations, which arise in many fields of science and technology (e.g., analysis of acoustic vibrations, oscillations in small molecules, optically torqued nanorods, Josephson junctions, electronic filters, gravitational lensing in general relativity, advanced models in field theory, oscillations of buildings during earthquakes, and others) [Lima F.M.S., 2008].

The nonlinear differential equation for the simple pendulum can be exactly solved and the period and periodic solution expressions involve the complete elliptic integral of the first kind and the Jacobi elliptic functions, respectively. Due to this, several approximation schemes have been developed to investigate the situation for large amplitude oscillations of a simple pendulum, and several approximations for its large-angle period have been suggested (a summary of most of them can be found in [Beléndez A., 2009]).

In 1997 M. I. Molina appears expression to pendulum equation from using interpolatory-like linearizations is shown for the simple pendulum which can be used for any initial amplitude [Molina M. I., 1997]. In 2002 Kidd and Fogg found that it is feasible to extend the theory to the case of larger amplitudes and to employ it in a fairly involved laboratory experiment [Kidd R. B., 2002]. In 2003 Millet proposed a mathematical justification for the Kidd and Fogg formula by considering trigonometric relation and small-angle approximations for sine and cosine functions, as well as the comparison between the sine function and various of its linear approximations [Millet L. E., 2003]. In 2005 Hite three approximations are discussed for the frequency of a simple pendulum. The first, proposed earlier by Kidd and Fogg, is simple in form but is the least accurate. The third, also based on the analogy to the simple harmonic oscillator, is also simple and about twice as accurate as the first. The second one is obtained using very little physics but is by far the most accurate [Hite G. E., 2005]. In 2006 Lima and 
Arun A simple approximate expression is derived for the dependence of the period of a simple pendulum on the amplitude. The approximation is more accurate than other simple relations. Good agreement with experimental data is verified [Lima F. M. S., 2006]. In 2007 Siboni studies the period of a pendulum can be accurately determined by an arithmetic-geometric map. The high efficiency of the map is due to super linear convergence [Siboni S., 2007]. In 2008 Amrani, et al. studies the experimental accuracy performance of each of the approximation expressions relative to the exact period for large amplitudes of a simple pendulum in the interval $0 \leq \theta \leq \pi$. The plots of the linearized exact period as a function of linearized formulae were carried out and relative errors in these expressions were investigated. Also, gives a clear idea how each formula approximates the exact period [Amrani D., 2008]. In 2008 Lima introduced a new approximate formula accurate for all amplitudes between 0 and $\pi$ rad. It is shown that this formula yields an error that tends to zero in both the small and large amplitude limits, a feature not found in any previous approximate formula [Lima F.M.S., 2008]. In 2009 Beléndez, et al. introduced an approximation scheme to obtain the period for large amplitude oscillations of a simple pendulum is analyzed and discussed. The analytical approximate formula for the period is the same that suggested by Hit, but it is now obtained analytically by means of a term-by-term comparison of the power-series expansion for the approximate period with the corresponding series for the exact period [Beléndez A., 2009]. In 2010 Beléndez, et al. used the Carvalhaes and Suppes approximate formula for deriving a simple and accurate solution for the pendulum equation of motion in terms of elementary functions. He also obtained a trigonometric approximation for the tension in the string whose maximum error is less than $0.27 \%$ for all values of the amplitude less than $\pi / 2$ rad [Beléndez A., 2010].

\section{The Pendulum Period}

A simple pendulum consists of a particle of mass $m$ hanging from an unstretchable, rigid massless string of length $\mathrm{L}$ fixed at a pivot point as shown in Fig. 1. The system freely oscillates in a vertical plane under the action of gravity. It is assumed that the motion is not affected by damping or external forcing, and motion occurs in a 2-dimensional plane, i.e. the bob does not trace an ellipse.

The differential equation which represents the motion of the pendulum is [Halliday D., 2004]:

$$
\frac{\mathrm{c} \cdot \theta}{\mathrm{de} t^{2}}+\frac{\bar{z}}{\ell} \sin \theta=0
$$

This is known as Mathieu's equation. It can be derived from the conservation of mechanical energy. At any point in its swing, the kinetic energy of the bob is equal to the gravitational potential energy it lost in falling from its highest position at the ends of its swing (the distance $(\ell-\ell \cos \theta)$ in the diagram). From the kinetic energy the velocity can be calculated.

The first integral of motion found by integrating (1) is

$$
\frac{\mathrm{d} \theta}{\mathrm{dt}}=\sqrt{\frac{2 \mathrm{~g}}{\ell}\left(\cos \theta-\cos \theta_{\mathrm{o}}\right)}
$$

It gives the angular velocity in terms of the angle and includes the initial displacement $\left(\theta_{0}\right)$ as an integration constant

The differential equation given above is not soluble in elementary functions. A further assumption, that the pendulum attains only small amplitude that is sufficient to allow the system to be solved approximately. Making the assumption of small angle allows the approximation to be made $\sin \theta \approx \theta$.

Substituting this approximation into eq. (1) yields the equation for a harmonic oscillator:

$$
\frac{\mathrm{d}^{2} 0}{\mathrm{dt}^{2}}+\frac{\mathrm{g}}{\mathrm{f}} \mathrm{\theta}=0
$$

Under the initial conditions $\theta(0)=\theta_{0}$ and $\mathrm{d} \theta / \operatorname{dt}(0)=0$, the solution is

$$
\theta(t)=\theta_{0} \cos \left(\sqrt{\frac{5}{\ell}} t\right), \quad \theta_{0} \lll 1
$$


Represent a simple harmonic motion where $\theta_{0}$ is the semi-amplitude of the oscillation (that is, the maximum angle between the string of the pendulum and the vertical). The period of the motion, the time for a complete oscillation (outward and return) is

$$
T_{0}=2 \pi \sqrt{\frac{l}{g}}, \quad \theta_{0} \ll 1
$$

which called Christiaan Huygens's law for the period. In this case, the period of oscillation depends on the length of the pendulum and the acceleration due to gravity, and is independent of the amplitude $\theta$.

\section{Exact period expression}

The differential equation modelling the free, undamped simple pendulum gives in eq. (1)

$$
\frac{d^{2} \theta}{d t^{2}}+\frac{g}{t} \sin \theta=0
$$

The oscillations of the pendulum are subjected to the initial conditions

$$
\varphi_{(0)}=\varphi_{0} \text { and } \omega_{0}=0
$$

where $\theta_{0}$ is the amplitude of oscillation. The system oscillates between symmetric limits $\left[-\theta_{0},+\theta_{0}\right]$. The periodic solution $\theta(\mathrm{t})$ of eq. (1) and the angular frequency $\omega$ (also with the period $\mathrm{T}=2 \pi / \omega)$ depend on the amplitude $\theta_{0}$.

Equation (1), although straightforward in appearance, is in fact rather difficult to solve because of the nonlinearity of the trigonometric function $\sin \theta$. There are no analytical solutions for the above differential equation. In fact, the solution is expressed in terms of elliptic integrals [Parwani R. R., 2004]. Hence, equation (1) is either solved numerically or various approximations are used. In the most simple of these approximations we consider that the angle $\theta$ is small, and then the function $\sin \theta$ can be approximated by $\theta$. Then the nonlinear differential equation (1) becomes a linear differential equation that can easily be solved, and the period $\mathrm{T}_{0}$ of the oscillation is given by

$$
T_{0}=2 \pi \sqrt{\frac{b}{g}}
$$

The period for this case is independent of the amplitude $\theta_{0}$ of oscillations and it is only a function of the length $\ell$ of the pendulum and the acceleration of gravity $\mathrm{g}$.

The exact value of the period of oscillations is given by the equation [Thornton S. T., 2004]:

$$
\mathrm{T}_{\mathrm{ex}}=\mathrm{T}_{0} \frac{2}{\pi} \mathrm{R}(\mathrm{k})
$$

Where $\mathrm{k}=\sin ^{2}\left(\theta_{0} / 2\right)$ and $\mathrm{K}(\mathrm{k})$ is the complete elliptic integral of the first kind. Its values have been tabulated for various values of $k$ can be shown in [Jeffrey A., 2008]. The power-series expansion of eq. (7) is [Fulcher L. P., 1976]

$$
T_{\mathrm{m} x} \approx T_{9}\left(1+\frac{1}{4} k^{2}+\frac{q}{b 4} k^{4}+\frac{25}{230} k^{6}+\cdots\right)
$$

Using the power-series expansion of $\mathrm{k}=\sin ^{2}\left(\theta_{0} / 2\right)$, we may write another series for the exact period

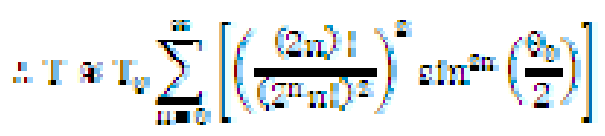

\section{The Experiment}

We used a Digital Camera (Type Sony) for Camera Movement simple pendulum back and forth where we started from the rest of ball and an angle of almost $15^{\circ}$; they arrived to the movement of the pendulum angle of 
less than $5^{\circ}$. After that, we converted the video clip in to still images (frame), and cut to 25 frames per second. Then we used the Segmentation technique to highlight the moving ball only then we easy find the angle at which you make with the vertical axis of the pendulum.

We measured the length of a pendulum from the center of the bob to the edge of a pendulum clamp and adjusted it to be as close to $0.20 \mathrm{~m}$. Figure (2) shows captured images of the pendulum Motion of in the laboratory.

Then we have found the angle of pendulum from compute the averages of $\mathrm{X}$-values and $\mathrm{Y}$-values for the indices of object image point. After that using the trigonometric functions to estimate the angle between vertical line and pendulum string in each image. By using Table Curve 2D software, we found at the period time through the relationship between the angular displacements with time and found the wavelength of the oscillating, which is the time of period. See Figure (3), which illustrates the period time in experiment of the multiple angles, where the time for the period represented by $(\mathrm{c} / 30)$.

\section{Comparison between exact and approximate expression}

We compare the accuracy of the approximation for the pendulum period in Eq. (9) to that of exact solution for amplitudes less than or equal to $\left(15^{\circ}\right)$.

Figure (4) illustrate the ratio between the actual period of a pendulum and the approximate value obtained for small angles, as a function of the amplitude.

Appears from (Fig. 4) the difference between a power series period time for the power 1 to power 5, also we see that the difference is increasing with increase the value of angle, it seems clear in the angles which more than $\left(20^{\circ}\right)$, while the values of the angle that is smaller than $\left(15^{\circ}\right)$ - The scope of work in this research - the difference does not appear clear from the values of the time, where the maximum difference in $\left(\theta=15^{\circ}\right)$ reach $(0.037 \mathrm{mSec})$, therefore, we will compare between the power series of power 5 with exact period time, which we got from the test.

The measurement of the time interval for several successive periods is a good strategy for oscillations in the small angle regime, where the amplitude does not change significantly from one swing to the next, but not for large angle oscillations, because the period decreases considerably due to air friction.

This behavior is confirmed in Figure (5), where the period $\mathrm{T}$ is plotted as a function of $\theta$. In Figure (5), Experimental data taken from Figure (3) also theoretical data taken from equation (9). The experimental data for amplitudes small than $\left(15^{\circ}\right)$ clearly reveal a systematic over estimation for the period due to air damping.

\section{Conclusion}

We found that it is feasible to extend the theory to the case of larger amplitudes and to employ it in a fairly involved laboratory experiment. As has been noted, the simple pendulum is particularly rich in physics implications, and an understanding of its behavior over a more realistic range of phenomena is a worthwhile goal.

It seems that the reported errors in approximating the exact period of the approximation formulae for large amplitudes of a simple pendulum in the range $0^{\circ} \leq \theta \leq 15^{\circ}$ are not very accurate for physics students who perform the simple pendulum experiment.

A simple approximate expression is derived for the dependence of the period of a simple pendulum on the amplitude. The approximation is more accurate than other simple relations. Good agreement with experimental data is verified.

\section{References}

Amrani D., Paradis P. and Beaudin M. (2008). Approximation expressions for the large-angle period of a simple pendulum revisited. Rev. Mex. Fis. E, 54, 59-64.

Baker G. L.and Blackburn J. A. (2005). The Pendulum: A Case Study in Physics (Oxford: Oxford University Press, 2005).

Beléndez A., Francés J., Ortuño M., Gallego S. and Bernabeu J.G. (2010). Higher accurate approximate solutions for the simple pendulum in terms of elementary functions, Eur. J. Phys., 31, 65-70.

Beléndez A., Rodes J.J., Beléndez T. and Hernández A. (2009). Approximation for the large-angle simple pendulum period, Eur. J. Phys., 30, 25-28.

Beléndez A., Rodes J.J., Beléndez T. and Hernández A. (2009). Approximation for the large-angle simple pendulum period, Eur. J. Phys, 30, 25-28. 
Fulcher L. P. and Davis B. F. (1976). Theoretical and experimental study of the motion of the simple pendulum, Am. J. Phys, 44, 51-55.

Halliday D., Resnick R., and Walker J. (2004). Fundamentals of Physics, $7^{\text {th }}$ ed. (Wiley, Hoboken, NJ).

Hite G. E. (2005). Approximations for the period of a simple pendulum, Phys. Teach, 43, 290-292.

Jeffrey A. and Dai H. (2008). Handbook of Mathematical Formulas and Integrals, $4^{\text {th }}$ ed. (Elsevier Inc.)

Kidd R. B. and Fogg S. L. (2002). A simple formula for the large-angle pendulum period, Phys. Teach, 40, 81-83.

Lima F. M. S. and Arun P. (2006). An accurate formula for the period of a simple pendulum oscillating beyond the small angle regime, Am. J. Phys, 74, 892-895.

Lima F.M.S. (2008). simple 'log formulae' for pendulum motion valid for any amplitude,” Eur. J. Phys., 29, 1091-1098.

Lima F.M.S. (2008). Simple 'log formulae' for pendulum motion valid for any amplitude, Eur. J. Phys, 29, 1091-1098.

Millet L. E. (2003). The large-angle pendulum period, Phys. Teach, 41, 162-163.

Molina M. I. (1997). Simple linearization of the simple pendulum for any amplitude, Phys. Teach., 35, 489-490.

Parwani R. R. (2004). An approximate expression for the large angle period of a simple pendulum, Eur. J. Phys, 25, 37-39.

Siboni S. (2007). Superlinearly convergent homogeneous maps and period of the pendulum, Am. J. Phys., 75, 368-373.

Thornton S. T. and Marion J. B. (2004). Classical Dynamics of Particles and Systems, 5th ed. (Brooks/Cole, New York)

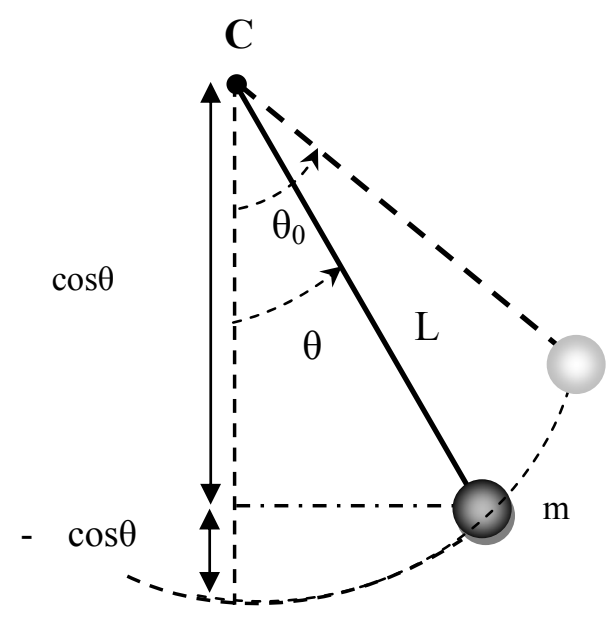

Figure 1. The simple pendulum 

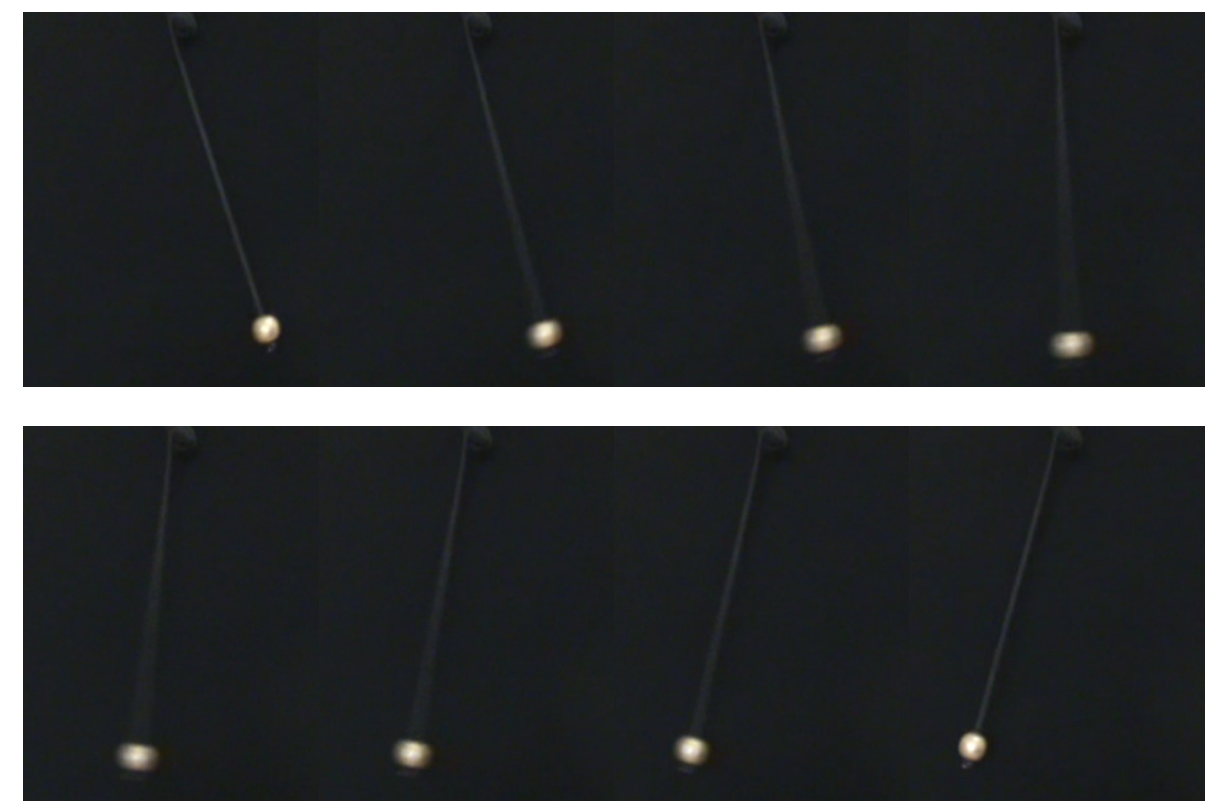

(a)
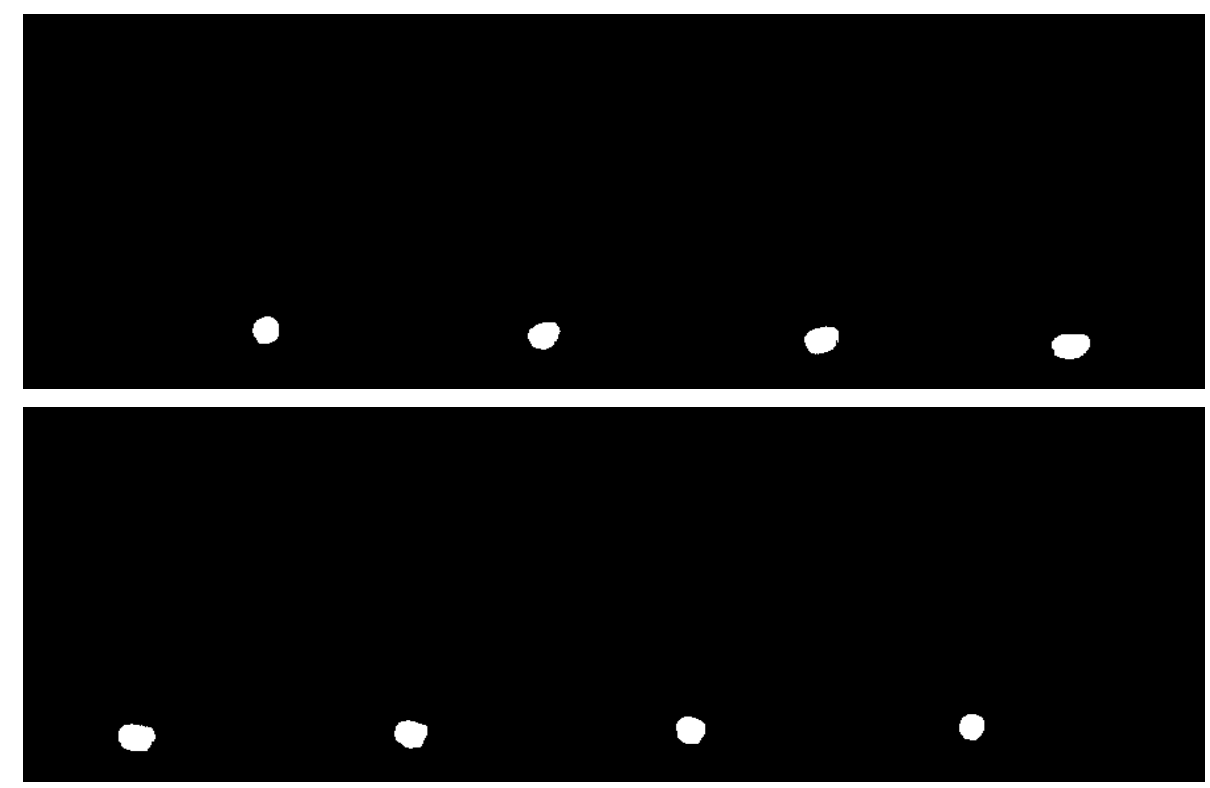

(b)

Figure 2. still images for pendulum moving

(a) Before segmentation. (b) After segmentation. 

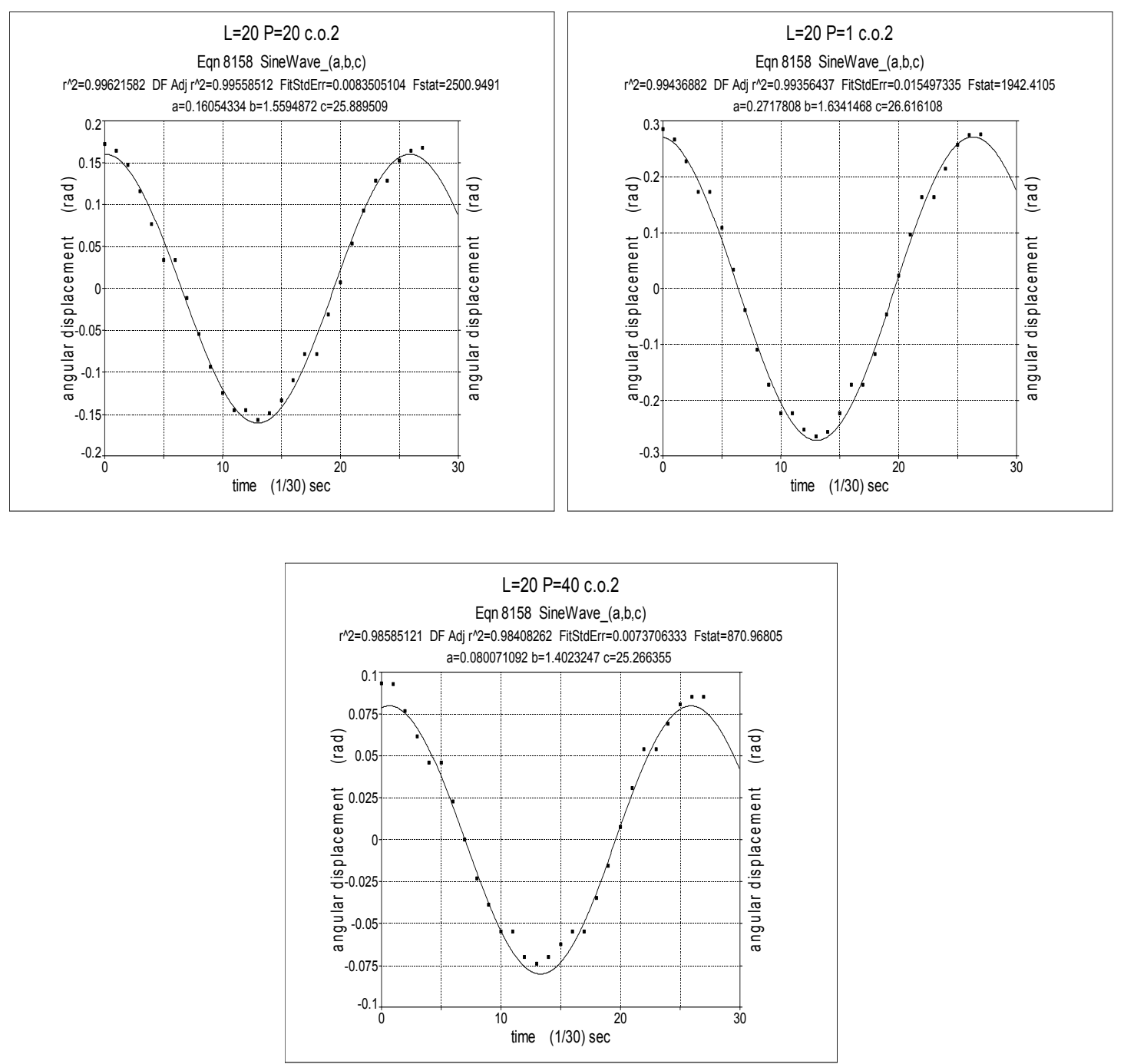

Figure 3. period of time for the multiple angles

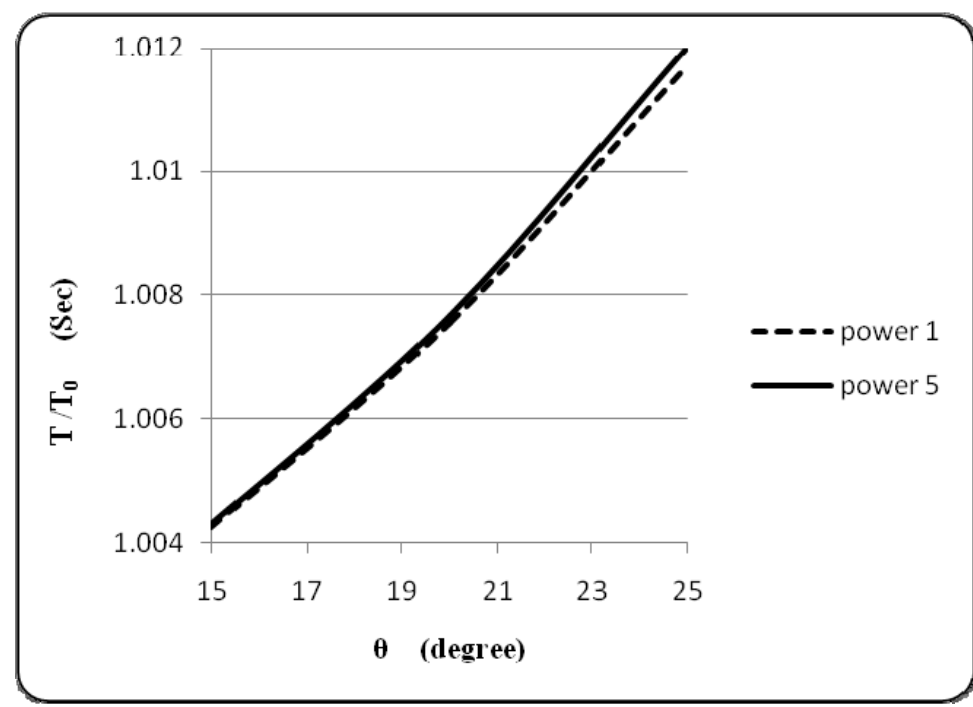

Figure 4. Deviation of the period from small-angle approximation 


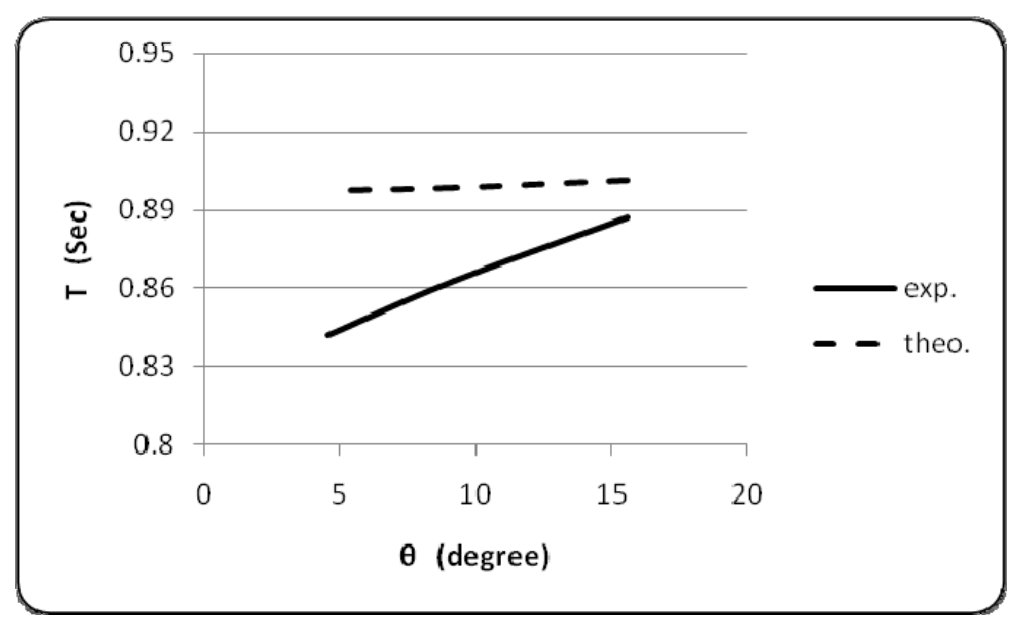

Figure 5. Experimental and theoretical period time 